malian cells the MAP kinase cascade may be regulated by both $\alpha$-subunits and $\beta \gamma$ dimers. Point mutations in $\alpha_{\mathrm{i} 2}$ which inhibit its GTPase (gip2 mutant) cause oncogenic transformation of some cells and activate MAP kinase ${ }^{7}$.

The new results ${ }^{4.5}$ show that the gip2 protein and other $\alpha$-subunits give only weak activation of MAP kinase in COS cells. But transfection of $\beta$ and $\gamma$ together is sufficient to activate MAP kinase, analogous to the action of these proteins (Ste $4 p$ and Ste18p) when overexpressed in $S$. cerevisiae. Both groups also show that heterologous expression of the $\alpha$-subunit of transducin in order to sequester $\beta \gamma$ dimers inhibits MAP kinase activation by co-transfected serpentine receptors (for example M1 or M2 carbachol receptors, D2 dopamine receptors and A1 adenosine receptors), but not by the endogenously expressed receptor for epidermal growth factor (EGF). So sequestration of free $\beta \gamma$ dimers, liberated by receptor-mediated trimer dissociation, selectively blocks the signalling of serpentine receptors to MAP kinase.

The icing on the cake is provided by Crespo et al. ${ }^{5}$, who show that the ability of $\beta \gamma$ to activate MAP kinase is inhibited by certain dominant-interfering Ras mutants; this suggests that $\beta \gamma$ uses Ras to activate MAP kinase. In this respect, the mammalian system differs from $S$. cerevisiae. Finally, Faure et al. ${ }^{4}$ address the issue of specificity by demonstrating that not all $\beta \gamma$ dimers are active in this system. This suggests that only receptors linked to a particular $\alpha \beta \gamma$ trimer combination may connect to Ras by this route.

How, then, are signals transmitted from $\beta \gamma$ dimers to Ras? Studies on the interaction of $\beta \gamma$ dimers with $\beta$-adrenergic receptor kinase ${ }^{\gamma}(\beta \mathrm{ARK}$, a negative regulator of the receptor involved in the adaptive response to hormone stimulation) provide some clues. $\beta \gamma$ dimers bind to the pleckstrin-homology $(\mathrm{PH})$ domain at the carboxy terminus of $\beta \mathrm{ARK}$, thereby activating the kinase domain ${ }^{8}$. The $\beta$ - and $\gamma$-subunits interact with each other through their amino termini and a model has been proposed in which the $\mathrm{PH}$ domain of $\beta$ ARK, $\beta$ and $\gamma$ may form a tripartite coiled coil, resulting in activation and translocation of $\beta \mathrm{ARK}$ to the membrane". The model remains speculative but provides us with a framework for considering how $\beta \gamma$ may connect to Ras. Thus, $\beta \gamma$ may bind to the $\mathrm{PH}$ domain of a Ras regulatory protein (the mammalian nucleotide-exchange factors, rasGRF and Sos, have PH domains, as does the GTPase-activating protein p120 GAP). Certainly $\beta \gamma$ dimers can bind to a glutathione $S$-transferase fusion protein consisting of the PH domain of rasGRF in vitro, though with reduced affinity compared to the binding to $\beta \mathrm{ARK}-\mathrm{PH}^{10}$ Reagents for testing these models are readily available, so we can expect answers before too long.

Alternative models can be envisaged. For example, the pathway connecting activation of the lysophosphatidic acid receptor to $R$ as is sensitive to certain kinase inhibitors ${ }^{2}$. The equivalent pathway in $S$. cerevisiae involves a kinase (Ste20p): analogous proteins may therefore be involved in mammalian cells. A mammalian homologue of STE 20 has been cloned, but it seems to be regulated by the $\mathrm{Cdc} 42$ family of Ras-related GTPases rather than by Ras itself ${ }^{11}$.

Comparison of yeast and mammalian systems, with respect to the mechanism by which $G$ proteins activate the MAP kinase cascade, has been less illuminating than might have been hoped. In S. cerevisiae, one such cascade is activated by $\beta \gamma$ in a Ras-independent fashion; in this organism Ras regulates adenylyl cyclase in response to nutrient status. In $S$. pombe a related cascade is activated by $\alpha$-subunits and Ras, apparently converging on the pathway in a co-dependent manner. The new results suggest that mammalian cells may use $\alpha$-subunits in a Ras-independent pathway (perhaps involving protein kinase $C$ ) and $\beta \gamma$ subunits in a Ras-dependent pathway. $\beta \gamma$ is also involved in adaptive responses to hormones in mammalian cells, whereas in $S$. cerevisiae this function may be carried out by $\alpha$. It is certainly remarkable that such structurally conserved biochemical entities are used in different ways, given the conservation elsewhere in the pathway (see figure and ref. 1).

When the details of the pathways are better understood, unifying themes may emerge. One such theme may be the recruitment of signalling molecules to the plasma membrane. In mammalian cells, the EGF receptor recruits Grb2/Sos to the plasma membrane to activate Ras using $\mathrm{SH} 2$ and $\mathrm{SH} 3$ domains; Ras is in turn involved in translocation of Raf to the plasma membrane, where the kinase somehow gets turned on. By analogy, $\beta \gamma$ may assemble a signalling complex at the membrane to activate Ras and other effector pathways by using $\mathrm{PH}$ domains to direct protein-protein interactions.

Simon Cook and Frank McCormick are at ONYX Pharmaceuticals, 3031 Research Drive, Richmond, California 94806, USA.

\footnotetext{
Egan. S. E. \& Weinberg. R. A. Nature 365, 781 (1993) 2. van Corven, E. J. etal. Proc. natn. Acad. Sci. U.S. A. 90 van Corven, E. J. etal.

3. Cook, S. J et al. EMBO J. 12, 3475-3485 (1993).

4. Faure. M., Voyno-Yasenetskaya, T. A. \& Bourne, H. R. J. biol. Chem. 269, 7851-7854 (1994)

Crespo, P. Xu, N. Simonds, W. F. \& Gutkind, J. S. Nature 369, 418-420 (1994)

Errede, B. \& Lewin, D. E. Curr. Opin. CellBiol.5. 254-260 (1993)

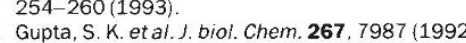

8. Ingiese, J. etal. J. biol. Chem. 268. 23735 (1993)

9. Simonds, W. F., Manji. H. K. \& Garritsen, A. Trends biochem. Sci. 18, 315-317 (1993).

10. Touhara, K. et al. J.biol. Chem. 269, 10217 (1994)

11. Manser, E. et al. Nature 367, 40-46 (1994)
}

\section{Butcher, baker}

BREAD, says Daedalus, is the most inspired of inventions. By a simple, even primitive process, a staple cereal grain is turned into food objects - loaves of bread with a pleasing identity, taste and structure. They can be produced in endless and subtle variety, stored and traded, enhanced with other foodstuffs, and used as raw material in further cookery. Best of all, bread is inherently so satisfying that you can eat it every day of your life and never tire of it.

Another fundamental foodstuff is the sausage. It has the humbler goal of repackaging a wide range of animal scraps, fragments of offal and similar proteinaceous discards. Daedalus is now combining the philosophies behind these two great culinary inventions.

$\mathrm{He}$ is developing a very basic way of reclaiming reject protein material. DREADCO's biochemists are taking various food wastes, offal, old hides, insect and rodent carcasses, bacterial biomass and so on, and grinding them up in water. Heat, pressure and protease enzymes can then chop up their protein chains into lengths short enough to be soluble. The resulting peptides can be neatly filtered from the ghastly mixture as a sterile solution. Their chains are then enzymatically recombined into a sort of randomized 'average protein'. It approaches the thermodynamically most stable combination of the original amino acids. It is a gluey, pasty mass, showing no trace of its dubious origins.

As a foodstuff, average protein is pretty uninspiring. The problem is that it lacks structure. Daedalus is seeking a blowing reaction to expand average protein into a foamed solid, and a vulcanizing agent to stabilize the resulting bread-like product by crosslinking its linear chains into a threedimensional molecular mesh. With luck, the yeast fermentation of added sugar will serve as a blowing reaction, as it does in baking bread. On later heating, the flavoursome Maillard protein-sugar reaction would then usefully develop the taste of the product. A dash of formaldehyde should harden and vulcanize it, though the result might resemble edible foamed Bakelite. A polyfunctional amino acid might do a better job.

The final product will be a splendid new mass-market foodstuff: a protein bread. It will be cheap, nutritious and satisfying, with a detailed texture and the sort of mild flavour that never palls, but enhances any foodstuff eaten with it. DREADCO's Marketing Department is already wondering what to call it. 'Meat Loaf' has the wrong connotations somehow.

David Jones 